ELECTROANALYTICAL CHEMISTRY AND INTERFACIAL ELECTROCHEMISTRY

Elsevier Sequoia S.A., Lausanne - Printed in The Netherlands

\title{
COMPLEX PLANE ANALYSIS OF SINGLE PULSE DATA
}

H. P. VAN LEEUWEN, D. J. KOOIJMAN, M. SLUYTERS-REHBACH AND J. H. SLUYTERS

Laboratory of Analytical Chemistry, State University Utrecht (The Netherlands)

(Received May 24th, 1969)

\section{INTRODUCTION}

The single pulse galvanostatic step method ${ }^{1}$ and the coulostatic impulse method $^{2,3}$ are two of the most powerful relaxation methods available for the study of electrode kinetics. Important advantages are the possibility of a precise compensation of the ohmic drop in the galvanostatic pulse method ${ }^{4}$ and the relative insensitivity to ohmic drop of the coulostatic pulse method, the large time scale that can be covered, and the fact that the overvoltage-time curve can be obtained in one experiment. In the last respect, the pulse methods compare especially favourably with the complex impedance method where variation of frequency, the equivalent of variation of time, requires numerous time-consuming measurements.

However, an analysis of experimental data indicates a disadvantage of the pulse methods compared with the impedance method because of the cumbersome expressions for the overvoltage-time relations. The expressions contain exp erfc functions of sometimes complex quantities. Only for short and large times are simpler approximations possible; these were used by Berzins and Delahay ${ }^{1}$ as the basis for an extrapolation procedure to determine the interfacial parameters. This procedure leaves, therefore, a considerable time region unused. The situation has been improved by Kooijman and Sluyters ${ }^{5}$ who proposed a numerical analysis procedure using nomograms. In these nomograms the properties of the overvoltage-time curve are precalculated in terms of dimensionless quantities on the basis of the classical theory, i.e. accounting for the presence of a purely capacitive double layer and an electrode reaction kinetically controlled by charge transfer and diffusional mass transfer. This method is advantageous for the detection of deviation from classical behaviour but a theory accounting for complications would probably be rather involved. Kooijman developed another $\operatorname{method}^{6}$, pointing out that at one electrolysis time, the overvoltage $\eta$ and its derivative with respect to time $t$, provide $t w o$ relations between the interfacial parameters. This resembles in a way the complex impedance method, where at one frequency the two components of the impedance are available. However, the determination of $\mathrm{d} \eta / \mathrm{d} t$ may be rather inaccurate.

A quite different approach has been made by Levart and Poirier d'Angé $\mathrm{d}^{\text {'Orsay }}{ }^{7,8}$ who elaborated on an idea introduced by Wijnen ${ }^{9}$, to make use of the Laplace transformation of experimental pulse data. It is proposed to determine the Laplace transforms of voltage and current for various values of the Laplace parameter $s$, by means of a graphical procedure. The quotient of the two transforms can be expressed as a function of $s$ with much simpler algebra than the overvoltage itself as 
a function of $t$. Moreover, the theory holds generally irrespective of the relaxation method under investigation. Expressions have been derived for the operational impedance on the basis of the classical theory ${ }^{7}$ and also for more sophisticated models including control by a homogeneous chemical reaction or reactant adsorption ${ }^{8}$.

There is a distinct resemblance between the theoretical form of the operational impedance as a function of $s$ and that of the "normal" impedance as a function of frequency, except that the latter is complex and delivers two data at one frequency. Recognizing this advantage, Levart and Poirier d'Angé d'Orsay ${ }^{8}$ suggested that a complex quantity $x+j y$ should be taken for $s$. They did not indicate, however, that this is not possible with a graphical integration procedure. Moreover, the expressions for the complex operational impedance are not as simple as those for the complex "normal" impedance. Essentially, the latter would result if $x=0 ; s=j y$ or $s=j \omega$ were therefore chosen. This could not be done in Levart's treatment because $x$ must be sufficiently large in order to meet the condition of convergence of the transform function.

In this paper we propose to describe the overvoltage-time curve as composed of a series of trial functions of which the Laplace transform is known mathematically. It will be shown that in this way the Laplace transform for any value of $s$, also imaginary, can be found. Consequently, it will be possible to analyze data of any pulse method following the procedures outlined for the complex plane analysis of impedances ${ }^{10}$.

THEORY

The Laplace transform, $f(s)$, of a time function, $f(t)$, is defined by ${ }^{11,12}$

$$
\bar{f}(s)=\int_{0}^{\infty} f(t) \mathrm{e}^{-s t} \mathrm{~d} t
$$

where $s$ is the operational Laplace parameter having a dimension $\mathrm{s}^{-1}$. An important property of the Laplace transformation is its linearity, which means that the transform of a linear combination of two or more functions equals a linear combination of the separate transforms of the functions:

$$
\overline{\Sigma c_{\mathrm{i}} f_{\mathrm{i}}(t)}=\Sigma c_{\mathrm{i}} \overline{f_{\mathrm{i}}(t)}
$$

It is well known that this transformation is a useful mathematical operation for finding the solution of a set of differential equations, e.g. Fick's laws in electrode reaction kinetics. Briefly, the differential equations are transformed following eqn. (1); with the appropriate boundary conditions, the transforms of current and voltage are found; finally, the desired current-voltage relation (e.g. for a.c. methods), the current-time relation (potentiostatic methods) or the overvoltage-time relation (galvanostatic and coulostatic methods) are derived by back-transformation after introduction of the properties of the method.

Another possibility introduced by Levart and Poirier d'Angé d'Orsay ${ }^{7,8}$, is to define the operational impedance $\bar{Z}(s)$ by:

$$
\bar{Z}(s)=\frac{\bar{V}(s)}{\bar{i}(s)}
$$

where $\bar{V}(s)$ and $i(s)$ are the transforms of voltage $V$ and current $i$. It has been shown ${ }^{7}$ 
that $\bar{Z}(s)$ can be derived as a function of $s$ without stating explicitly what kind of relaxation method is employed. Therefore, if for a given method it is possible to determine the Laplace transform for various $s$-values of the experimental current and voltage, it is possible to analyze the data with the general (universal) expression for $\bar{Z}(s)$. Also, it would be possible to predict from the experimental data obtained with one method (say, the galvanostatic method), the results that would be obtained with another method, simply by back-transformation for that particular method.

A special advantage of the impedance method is that back-transformation is performed simply by taking $s=j \omega$. This is shown by the following reasoning. Let the current $i$ be given by:

$$
i=i_{\mathrm{m}} \sin \omega t
$$

The complex impedance

$$
Z(\omega)=Z^{\prime}-j Z^{\prime \prime}
$$

is defined in such a way that

$$
V=i_{\mathrm{m}}\left[Z^{\prime} \sin \omega t-Z^{\prime \prime} \cos \omega t\right]
$$

With eqn. (1) it is easily calculated that

$$
\begin{aligned}
& \overline{\sin \omega t}=\frac{\omega}{\omega^{2}+s^{2}} \\
& \overline{\cos \omega t}=\frac{s}{\omega^{2}+s^{2}}
\end{aligned}
$$

The operational. impedance becomes according to eqns. (2)-(7)

and

$$
\bar{Z}(s)=Z^{\prime}-\frac{s}{\omega} Z^{\prime \prime}
$$

$$
\bar{Z}(j \omega)=Z^{\prime}-j Z^{\prime \prime}
$$

Concluding, the impedance of a circuit can be predicted from data obtained with other (e.g. pulse) methods, if it is possible to determine the Laplace-transform of these data for various imaginary values of $s=j \omega$.

Thus, in fact we are concerned with the transformation

$$
f(j \omega)=\int_{0}^{\infty} f(t) \mathrm{e}^{-j \omega t} \mathrm{~d} t
$$

for both current and voltage in the galvanostatic and coulostatic method. As $f(t)$ is zero for $t<0$ (which is the case in the pulse methods considered) a transformation according to eqn. (9) becomes identical with the well-known Fourier transformation, where originally the limits are $-\infty$ and $+\infty$ instead of 0 and $+\infty$ (one-sided Fourier integral).

In the coulostatic method, the voltage response $\eta$ decreases with time, so the transformation integral in eqn. (8) converges, which is evidently required:

$$
\lim _{t \rightarrow \infty}\left[\eta(t) \mathrm{e}^{-j \omega t}\right]=0
$$


Consequently, the transform can be computed directly writing eqn. (9) in the form:

$$
\bar{\eta}(j \omega)=\int_{0}^{\infty} \eta(t)[\cos \omega t-j \sin \omega t] \mathrm{d} t
$$

The coulostatic input signal, a very short current impulse, may be described in terms of the Dirac delta function ${ }^{3}$, i.e.

$$
i(t)=q \delta(t)
$$

where $q$ is the change in charge density on the electrode as a consequence of the current pulse, which is assumed to be entirely consumed by the charging of the double layer. The Laplace transform of a delta function is unity ${ }^{3}$, and the desired current transform is therefore

$$
i(j \omega)=\int_{0}^{\infty} q \delta(t) \mathrm{e}^{-j \omega t} \mathrm{~d} t=q
$$

The impedance of the circuit under investigation can thus be "determined" from coulostatic pulse data by computing $\tilde{\eta}(j \omega)$ numerically or graphically following eqn. (10) and dividing this by $q$.

In the galvanostatic step method, a current impulse represented by a step function is passed through the circuit and the voltage response for a galvanic cell is known to increase with time. For large values of $t$, i.e. in the diffusion-controlled domain, the increase is linear with $\sqrt{t}$ :

$$
\eta(t)=b+c \sqrt{ } t
$$

Thus, both current and voltage transform following eqn. (9) cannot be obtained directly because they do not meet the requirement of convergence. However, if we consider a transformation of $\eta(t)$ following eqn. (1) with a complex Laplace parameter

$$
s=\Delta+j \omega,
$$

the situation becomes completely different. Because $e^{-\Delta t}$ decreases exponentially with time, and $c \sqrt{ } t$ increases with time but is of lower order, the condition of convergence is fulfilled, even if $\Delta$ approaches zero:

$$
\lim _{\substack{t \rightarrow \infty \\ \Delta \rightarrow 0}}\left[\eta(t) \mathrm{e}^{-(\Delta+j \omega) t}\right]=0
$$

For the constant galvanostatic current, the same argument holds. Although we now have to do with transforms that are functions of both $\Delta$ and $j \omega$, we will show that calculation of $Z(j \omega)$ as defined by eqn. (3) is still possible, because as a correct approximation $\Delta$ can be neglected in the quotient of voltage and current transforms:

$$
\lim _{\Delta \rightarrow 0} \frac{\int_{0}^{\infty} c \sqrt{t} \mathrm{e}^{-(\Delta+j \omega) t} \mathrm{~d} t}{\int_{0}^{\infty} I e^{-(\Delta+j \omega) t} \mathrm{~d} t}=\lim _{\Delta \rightarrow 0} \frac{c(\Delta+j \omega)^{-\frac{3}{2}}}{I(\Delta+j \omega)^{-1}}=\frac{c \omega^{-\frac{1}{2}}}{I \sqrt{ } 2}-j \frac{c \omega^{-\frac{1}{2}}}{I \sqrt{2}}
$$

This result is identical with what would be obtained directly from the transformation 
eqn. (9). The denominator of the operational impedance can therefore be computed for $s=j \omega$ as $I / j \omega$, if $I$ represents the magnitude of the galvanostatic current density.

However, there is still the difficulty that the $\eta-t$ curve, which is an experimental result, cannot be transformed directly with $s=j \omega$. To meet this objection, an artifice must be applied in the sense that the $\eta-t$ curve is described by means of an algebraic equation consisting of one or more functions of which the Laplace transform is mathematically known. This equation can be found by choosing a number of trial functions which in a linear combination are, as closely as possible, in agreement with the experimental data. A least-squares procedure determines the best combination, i.e. the coefficients of the trial functions:

$$
\eta(t)=\sum_{i=1}^{n} c_{\mathrm{i}} f_{\mathrm{i}}(t)
$$

In principle, the number of trial functions to be used may be chosen freely, but the character of the functions is, naturally, dependent on the $\eta-t$ curve. As we wanted to find a universally useful series of functions, some $\eta-t$ curves calculated for different types of electrode reactions, i.e., different sets of the interfacial parameters describing classical behaviour ${ }^{5}$, were analyzed using various combinations of various trial functions. For galvanostatic curves the following system, strongly inspired by the theoretical $\eta-t$ equation, was found to be satisfactory:

$$
\begin{array}{r}
\frac{c_{1}}{\gamma_{1}-\beta_{1}}\left\{\frac{\gamma_{1}}{\beta_{1}^{2}}\left[\exp \left(\beta_{1}^{2} t\right) \operatorname{erfc}\left(\beta_{1} \sqrt{ } t\right)+2 \beta_{1}\left(\frac{t}{\pi}\right)^{\frac{1}{2}}-1\right]-\right. \\
\left.\frac{\beta_{1}}{\gamma_{1}^{2}}\left[\exp \left(\gamma_{1}^{2} t\right) \operatorname{erfc}\left(\gamma_{1} \sqrt{ } t\right)+2 \gamma_{1}\left(\frac{t}{\pi}\right)^{\frac{1}{2}}-1\right]\right\}+ \\
\frac{c_{2}}{\gamma_{2}-\beta_{2}}\left\{\frac{\gamma_{2}}{\beta_{2}^{2}}\left[\exp \left(\beta_{2}^{2} t\right) \operatorname{erfc}\left(\beta_{2} \sqrt{ } t\right)+2 \beta_{2}\left(\frac{t}{\pi}\right)^{\frac{1}{2}}-1\right]-\right. \\
\left.\frac{\beta_{2}}{\gamma_{2}^{2}}\left[\exp \left(\gamma_{2}^{2} t\right) \operatorname{erfc}\left(\gamma_{2} \sqrt{ } t\right)+2 \gamma_{2}\left(\frac{t}{\pi}\right)^{\frac{1}{2}}-1\right]\right\}
\end{array}
$$

in which $c_{1}$ and $c_{2}$ denote the coefficients that are determined by the least-squares procedure and $\beta_{1}, \gamma_{1}, \beta_{2}$ and $\gamma_{2}$ are chosen variables which are allowed to be complex.

The Laplace transforms of the two similar functions have been given by Berzins and Delahay ${ }^{1}$. Division of these transforms by $I / s$ gives in the first instance the operational impedance $Z(s)$. After substitution of $s=j \omega$ and rearrangment, the complex impedance $Z(\omega)$ is found as an algebraic function of $\omega$, for which, of course, various values can be inserted.

Although, as pointed out before, the coulostatic curves could be transformed by a direct numerical calculation, it was found preferable to develop also for the coulostatic method a procedure with trial functions. Excellent results were obtained with the following combinations of trial functions:

$$
\begin{aligned}
& c_{1} \exp \left(A^{2} t\right) \operatorname{erfc}(A \sqrt{ } t)+c_{2} \exp \left(B^{2} t\right) \operatorname{erfc}(B \sqrt{ } t)+ \\
& c_{3} \exp \left(C^{2} t\right) \operatorname{erfc}(C \sqrt{ } t)+c_{4} \exp (-D t)+c_{5} \exp (-E t)
\end{aligned}
$$

where $c_{1}-c_{5}$ again represent the coefficients, and $A, B, C, D$, and $E$ are chosen quantities which are real in this case. The overvoltage transform $\bar{\eta}(j \omega)$ is here found as the 
sum of the five separate Laplace transforms, taken from Churchill ${ }^{11}, s$ being replaced by $j \omega$. Division by the coulostatic current transform, $q$ (see eqn. (11)), gives $Z(j \omega)$.

Once $Z(j \omega)$ is obtained, the further analysis can be performed following the procedures of the complex plane method ${ }^{10}$. As a special advantage, in both pulse methods the ohmic resistance does not contribute to the overvoltage, so that in fact the interfacial impedance $Z_{\mathrm{el}}$ is obtained. As the analysis procedures always start with the interfacial admittance $Y_{\mathrm{el}}=1 / Z_{\mathrm{el}}$, it is preferable to take the reciprocal of $Z(j \omega)$ and to split up this into its real and imaginary parts $Y^{\prime}(\omega)$ and $Y^{\prime \prime}(\omega)$. The frequency-dependence of these data can then be analyzed as outlined previously ${ }^{10,13}$.

The application of the method in practice will be demonstrated with the $\mathrm{Cd}^{2+} / \mathrm{Cd}(\mathrm{Hg})$ electrode reaction in $1 \mathrm{M} \mathrm{Na}_{2} \mathrm{SO}_{4}$ as an example.

\section{EXPERIMENTAL}

The electrical set-up for the galvanostatic and coulostatic measurements has been described previously ${ }^{4}$. The cell was placed in a copper box to reduce the pick-up of disturbing signals, and thermostatted at $25^{\circ} \mathrm{C}$. The cell-solution contained $5 \mathrm{mM}$ $\mathrm{Cd}^{2+}$ and $1 \mathrm{M} \mathrm{Na}_{2} \mathrm{SO}_{4}$, acidified with $\mathrm{H}_{2} \mathrm{SO}_{4}$ to $\mathrm{pH} 4$. Oxygen was removed by means of tank nitrogen. The electrode material, $5 \mathrm{~m} M \mathrm{Cd}$ amalgam, was prepared electrolytically and kept in a nitrogen atmosphere. Two amalgam drops served as working electrodes; the advantages of this arrangement have been mentioned previously ${ }^{14}$. The drops were refreshed before each measurement and the discarded drops were diluted in a pool of mercury to prevent dissolution of cadmium.

\section{RESULTS AND DISCUSSION}

\section{Classical method}

The system $\mathrm{Cd}^{2+} / \mathrm{Cd}(\mathrm{Hg})$ in $1 \mathrm{M} \mathrm{Na}_{2} \mathrm{SO}_{4}$, has already been studied with the galvanostatic single-pulse method ${ }^{15}$. The values, $D_{\mathrm{O}}=5.3 \times 10^{-6}$ and $D_{\mathrm{R}}=11 \times 10^{-6}$ for the diffusion coefficients, and $k_{\mathrm{sh}}=6.3 \times 10^{-2}$ for the rate constant were found.

TABLE 1

DATA ON THE $\mathrm{Cd}^{2+} / \mathrm{Cd}(\mathrm{Hg})$ ELECTRODE IN $1 \mathrm{M} \mathrm{Na}_{2} \mathrm{SO}_{4}$ AT $\mathrm{pH}=4 . \mathrm{Cd}^{2+}=5 \mathrm{~m} M, \mathrm{Cd}(\mathrm{Hg})=5 \mathrm{~m} M$

\begin{tabular}{|c|c|c|c|}
\hline & $\begin{array}{l}\left(1 / C_{\mathrm{O}} D_{\mathrm{O}}^{\frac{1}{2}}+1 / C_{\mathrm{R}} D_{\mathrm{R}}^{\frac{1}{\mathrm{R}}}\right) / \\
10^{7} \mathrm{~mol}^{-1} \mathrm{~cm} \mathrm{~s}^{\frac{1}{2}}\end{array}$ & $i_{0} / m A c^{-2}$ & $C_{\mathrm{d}} / \mu F \mathrm{~cm}^{-2}$ \\
\hline Galvanostatıc $\int$ this method & 16 & 65 & 19 \\
\hline \{classical way & 16 & 68 & 19 \\
\hline Coulostatic $\int$ this method & 16 & 58 & 21 \\
\hline \{classical way & - & 54 & 21.5 \\
\hline Literature values ${ }^{15}$ & 15 & 61 & 20 \\
\hline
\end{tabular}

The quantities listed in Table 1 (literature values) were calculated with these values. For the double layer capacity, a mean value $C_{\mathrm{d}}=20 \mu \mathrm{F} \mathrm{cm}^{-2}$ was reported ${ }^{5}$. It can be seen in Table 1 that our values, obtained in the classical way, are in good agreement with the literature values. However, the coulostatic data are insufficiently controlled by diffusion to produce the diffusional parameter. 


\section{Transform method}

The calculations were performed with a computer (ElX8) and the program, written in Algol, contained sub-routine programs for

(i) evaluation of the exp erfc, for both real and complex argument ${ }^{16}$;

(ii) determination of the least-squares solution of a linear system of equations;

(iii) calculation of $Z^{\prime}, Z^{\prime \prime}, Y^{\prime}$ and $Y^{\prime \prime}$.

Curves are calculated according to eqns. (16) and (17) for various combinations of the variables. The mean quadratic error - with respect to the experimental $\eta-t$ curveis used as a quality standard for the fitting of the calculated curve. The condition was made that the calculated and experimental curves should fit within the accuracy of the experimental data, which is $2 \%$ for longer times and up to $5 \%$ for shorter times, the latter owing to mis-compensation of the ohmic drop. This condition is reasonable, because the errors in the experimental data are more or less eliminated by the leastsquares fitting procedure.

The electrode admittance components obtained as a function of frequency were analyzed according to the complex plane method ${ }^{10}$, i.e. the curve of $Y_{\mathrm{e} 1}^{\prime} v$ s. $\sqrt{ } \omega$ was fitted to the best combination of values for the Warburg coefficient $\sigma$ and the irreversibility quotient $p^{\prime}$. The value of $C_{\mathrm{d}}$ was then calculated from $Y_{\mathrm{el}}^{\prime \prime}, \sigma$ and $p^{\prime}$.

The agreement with the data earlier obtained, see Table 1, is excellent. It is remarkable that even from coulostatic data a very good diffusional parameter is computed. It must be admitted, however, that this value is much less accurate than the others because in the $\eta-t$ curve there is little control by diffusion. This point will be discussed further below.

\section{Frequency region}

When the expression for the complex impedance $Z(j \omega)$ is obtained, one might, in principle, fill in any value of $\omega$. However, not every result makes sense because $\omega$ is associated with the original variable, the electrolysis time $t$. From the definition of the applied transformation, eqn. (1), it is easily recognized that if $s$ is much smaller than the reciprocal of the electrolysis time $t_{\max }$, too large a part of the integral corresponds to the time beyond $t_{\max }$, where, of course, $\eta$ is not known. There is also an upper limit for $s$ and thus for $\omega$, since during a certain time $t_{\min }$ from the start of the electrolysis, the $\eta-t$ curve is not known exactly because of transients. It is reasonable, therefore, to accept as "real" frequencies the values in the domain between $1 / t_{\max }$ and $1 / t_{\min }$. This means for the galvanostatic method, $200<\omega<10^{6}$. In the coulostatic method the lower frequency range will be higher.

On the other hand, the trial functions in (16) and (17) have been chosen in such a way that the computed $\eta-t$ equation always obeys eqn. (6) at long times. This means that the electrode process is assumed to be controlled entirely by diffusion. Extension of the frequency domain to frequencies smaller than $1 / t_{\max }$ can therefore be considered as an extrapolation to zero frequency from the lower part of the "real" frequency domain. This extension, which gives optimal information concerning the diffusional parameter, is made possible by the integration in eqn. (9) or, in fact, eqn. (1), which takes into account the time domain from zero to infinity, whereas the measurement corresponds to times no larger than a few milliseconds.

Levart and Poirier d'Angé d'Orsay claimed in their second paper that a frequency range from zero up to a few $\mathrm{MHz}$ can be attained. We consider this upper 
limit too high, because it would mean that overvoltages have to be measured in the $0.1 \mu$ s range, which so far has not been realized.

Direct transformation of the coulostatic response

As stated before, the coulostatic $\eta-t$ curve could be transformed without the help of trial functions by multiplication of $\eta(t)$ by $(\cos \omega t-j \sin \omega t)$ and integration of this following eqn. (10). Division by $q$, according to eqn. (12), delivers $Z^{\prime}-j Z^{\prime \prime}$ as a function of $\omega$. We did not apply this method because of the rather easy success we obtained with the trial function method. However, it must be noted that so far we have considered only electrode reactions with classical behaviour, i.e. systems in accordance with the Randles circuit ${ }^{10}$. For other systems (e.g. involving chemical reactions, reactant adsorption or other surface phenomena) quite different trial functions might be needed. This difficulty is avoided in the direct method as mentioned here.

The calculations could be performed numerically with the aid of a computer. It is also possible with an analog computer to multiply and integrate electronically, so that after division by $q, Z^{\prime}$ and $Z^{\prime \prime}$ can be read out directly.

CONCLUSION

The "complex plane analysis of pulse data" proposed can be compared with the former analysis procedures of pulse methods on the one hand and with the complex impedance method on the other. With respect to the latter, it can be concluded that a large frequency range is covered by the new method (up to at least $100 \mathrm{kHz}$ instead of $10 \mathrm{kHz}$ in the impedance method). As a consequence, faster electrode reactions and other high-frequency phenomena can be studied. The whole frequency-region is optimally used because the elegant analysis procedures of the complex plane method can be used. The accuracy of the method is completely determined by the experimental precision, which in the lower frequency range is worse than with bridge measurements of the impedance, but certainly not at the higher frequencies. Finally, it remains spectacular that the electrode impedance or admittance at an (in principle) infinite number of frequencies is obtained by one measurement which lasts no longer than a few milliseconds.

\section{ACKNOWLEDGEMENTS}

The authors wish to express their gratitude to Dr. B. Timmer for valuable discussions and to Drs. C. A. Ch. Görts for placing the least-squares sub-routine program at their disposal.

The present investigations have been carried out in part under the auspices of the Netherlands Foundation of Chemical Research (S.O.N.) with financial aid from the Netherlands Organization for the Advancement of Pure Research (Z.W.O.).

SUMMARY

The evaluation of kinetic parameters from galvanostatic and coulostatic step measurements is performed in a new way. The experimental overvoltage/time curve 
is written as an analytical function and transformed into the Laplace domain. This implicates that the variable time has been replaced by the operational frequency, $s$, which is given a completely imaginary value, $j \omega$. Both the real and imaginary components of the complex electrode impedance can be easily computed for any chosen value of the angular frequency, $\omega$. Subsequent analysis according to the complex plane method delivers the desired information. The procedure is illustrated by application to data obtained with the $\mathrm{Cd}^{2+} / \mathrm{Cd}(\mathrm{Hg})$ electrode in $1 \mathrm{M} \mathrm{Na}_{2} \mathrm{SO}_{4}$ at $\mathrm{pH} 4$.

\section{REFERENCES}

1 T. Berzins and P. Delahay, J. Am. Chem. Soc., 77 (1955) 6448.

2 P. Delahay, J. Phys. Chem., 66 (1962) 2204.

3 W. H. Reinmuth, Anal. Chem., 34 (1962) 1272.

4 D. J. Kooljman and J. H. Sluyters, Electrochim. Acta, 11 (1966) 1147.

5 D. J. Koouman and J. H. Sluyters, Electrochim. Acta, 12 (1967) 1579.

6 D. J. Kooljman, J. Electroanal. Chem., 18 (1968) 81.

7 E. Levart and E. Poirier d'Angé D'Orsay, J. Electroanal. Chem., 12 (1966) 277.

8 E. Levart and E. Poirier d'Angé d'Orsay, J. Electroanal. Chem., 19 (1968) 335.

9 M. D. WiJnen, Rec. Trav. Chim., 79 (1960) 1203.

10 M. Sluyters-Rehbach and J. H. SluYters in A. J. Bard, (Ed.), Electroanalytical Chemistry, Marcel Dekker, New York, in press.

11 R. V. Churchill, Operational Mathematics, McGraw-Hill, New York, 1958.

12 W. D. DAY, Introduction to Laplace Transforms, Iliffe, London, Interscience Publ., 1960.

13 B. Timmer, M. Sluyters-Rehbach and J. H. Sluyters, J. Electroanal. Chem., 18 (1968) 93.

14 D. J. Kooluman, M. Sluyters-Rehbach AND J. H. SluYters, Electrochim. Acta, 11 (1966) 1197.

15 D. J. KoOUman and J. H. SluYTERS, Electrochim. Acta, 12 (1967) 693.

16 V. N. Faddeyeva And N. M. Terent'ev, Tables of Values of the Exponential Error Function Complement for Complex Argument, Pergamon Press, New York, 1961. 